\title{
Intensifying Ebola treatment in Africa to reduce high mortality rate
}

\author{
n Cite as: CMAJ 2019 April 23;191:E458-9. doi: 10.1503/cmaj.109-5734
}

Posted on cmajnews.com on Apr. 4, 2019.

W

ith over $60 \%$ of patients stricken with Ebola dying in the recent outbreak in the Democratic Republic of the Congo, some Canadian critical care doctors are arguing for more aggressive care, including renal dialysis and mechanical ventilation. That would bring the level of treatment closer to that provided to 27 expatriates infected in West Africa who received care at hospitals in wealthy countries during the 2014-16 Ebola outbreak.

"Effectively, there were no magic bullets in Europe and North America for the expats that were evacuated and their mortality was $18 \%$," said Dr. Rob Fowler, a critical care doctor at Toronto's Sunnybrook Health Sciences Centre who coauthored an editorial on the subject in The Lancet. He treated patients with Ebola in West Africa as an advisor to the World Health Organization (WHO) and was in the Congo for the WHO last fall.

The Congo's northeast corner, where Ebola is spreading, may seem an unlikely place to push for critical care. It's an area where families struggle with malnutrition and malaria. Personal security is also a problem, because of a longstanding conflict among several warring groups that's placed those responding to Ebola in danger.

And not everyone accepts the group's ideas. Dr. Armand Sprecher, an Ebola expert with Médecins sans Frontieres (MSF), is concerned that "bringing critical care to bear on a few patients would likely impair our ability to do what we should be doing for the other ones."

But Dr. François Lamontagne, a critical care physician at the Centre hospit-

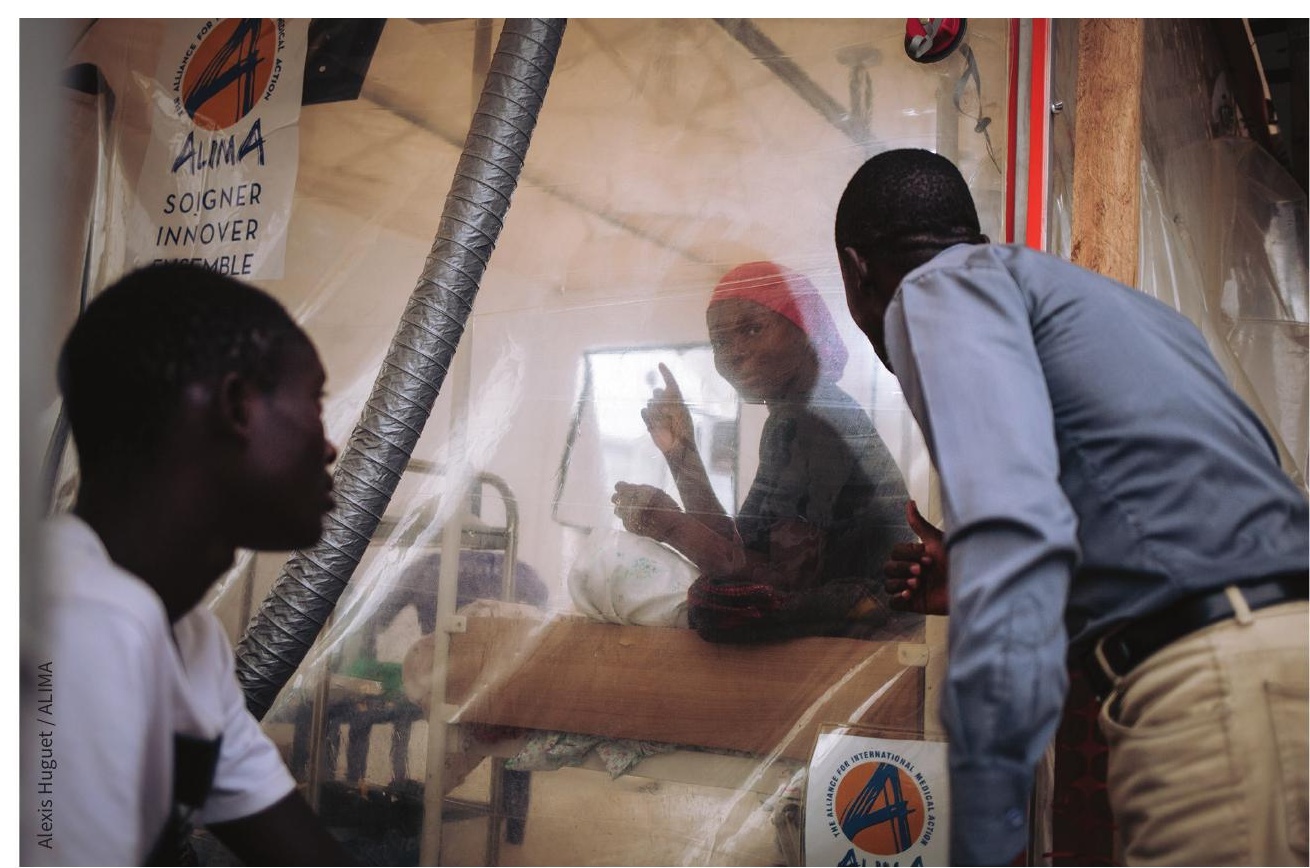

A woman being treated for Ebola inside a CUBE treatment centre in the Congo.

alier universitaire de Sherbrooke, and another of the editorial's coauthors, said he and his colleagues are accustomed to pushing against skeptics when it comes to Ebola care. He was outspoken about the need for intravenous fluids to treat Ebola in West Africa in 2014. At the time, some saw the procedure as impossible because of lack of infrastructure. Frustrated, he wrote a commentary with Fowler and others in the New England Journal of Medicine that compared hands-off treatment of patients with Ebola to denying life-supporting therapies to people with HIV. A few months later, he and others published data in the same journal suggesting that more aggressive care might have resulted in a lower mortality rate.
When the outbreak ended in 2016, Lamontagne led an effort to produce an evidence-based guideline for Ebola treatment. Published in 2017, the guideline calls for intravenous fluids as needed, and those providing treatment in the Congo today generally follow it. Trish Newport, a Canadian nurse with MSF who recently returned from the Congo, said that in a big change from how the organization delivered care in West Africa, MSF staff in the Congo give intravenous fluids and medications if needed, "because if people are really sick, they can't take anything orally."

Since August, patients in the Congo with Ebola have also been offered one of four experimental drugs through a clinical trial the country is running with the 
National Institutes of Health, or through a protocol the WHO developed for compassionate use of the drugs. But the medicines are more effective when taken early in the course of the illness, and many patients with Ebola in the Congo are arriving for treatment with high fever, vomiting and diarrhea already.

Fowler thinks patients with Ebola who are sick should get a peripherally inserted central intravenous line to allow for faster rehydration and treatment of electrolyte abnormalities. Though standard in Canadian intensive care units, central lines are rare in the Congo's Ebola treatment units. Fowler was occasionally able to insert peripheral central lines in the Congo while working at the CUBE (Chambre d'Urgence Biosécurisée pour Epidémies), a novel Ebola treatment facility that enables doc- tors to deliver some forms of care without donning full protective gear.

The CUBE was constructed by the Alliance for International Medical Action. The group's president, Dr. Richard Kojan, is also advocating for a higher level of care. He said health care workers in the CUBE are already doing blood transfusions when necessary, since Ebola can cause internal bleeding and Congolese authorities approved access to a blood bank. Of 26 patients with Ebola transfused in the CUBE by mid-March, 11 survived, Kojan said.

Since patients with Ebola often go into renal failure, he wants to offer dialysis in the form commonly used in intensive care units: continuous renal replacement therapy. The Congo health authorities gave Kojan a green light. Lamontagne, who is also working on the project, said the plan is to set up a sham version of the CUBE in West Africa, where nurses and doctors can determine the ideal way to deliver the therapy before bringing renal replacement to the Congo.

In Toronto, Fowler and critical care physician Dr. Neill Adhikari will be observing volunteer health care workers in personal protective equipment perform sham renal replacement and sham mechanical ventilation in experiments at a mock Ebola treatment unit at Defence Research and Development Canada's Toronto Research Centre. The research aims to measure the feasibility and risk of delivering that level of care in protective gear.

Miriam Shuchman, Toronto, Ontario 